\title{
Assessment of lung function in a large cohort of patients with acromegaly
}

\author{
Sylvère Störmann ${ }^{1}$, Bodo Gutt' ${ }^{2}$, Josefine Roemmler-Zehrer', Martin Bidlingmaier', \\ Rudolf M Huber ${ }^{3}$, Jochen Schopohl' ${ }^{1}$ and Matthias W Angstwurm ${ }^{1}$ \\ ${ }^{1}$ Medizinische Klinik und Poliklinik IV, Klinikum der Universität München und Lungentumorzentrum München, \\ Germany, ${ }^{2}$ Asklepios Klinik Birkenwerder, Abteilung für Diabetologie, Birkenwerder, Germany, and ${ }^{3}$ Sektion \\ Pneumologie Innenstadt und Thorakale Onkologie, Medizinische Klinik und Poliklinik V, Klinikum der Universität \\ München und Lungentumorzentrum München, Germany
}

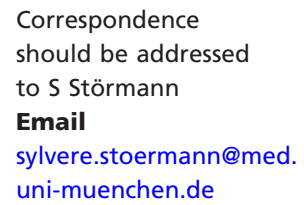

\begin{abstract}
Objective: Acromegaly is associated with increased mortality due to respiratory disease. To date, lung function in patients with acromegaly has only been assessed in small studies, with contradicting results. We assessed lung function parameters in a large cohort of patients with acromegaly.

Design: Lung function of acromegaly patients was prospectively assessed using spirometry, blood gas analysis and body plethysmography. Biochemical indicators of acromegaly were assessed through measurement of growth hormone and IGF-I levels. This study was performed at the endocrinology outpatient clinic of a tertiary referral center in Germany.

Methods: We prospectively tested lung function of 109 acromegaly patients (53 male, 56 female; aged 24-82 years; 80 with active acromegaly) without severe acute or chronic pulmonary disease. We compared lung volume, air flow, airway resistance and blood gases to normative data.

Results: Acromegaly patients had greater lung volumes (maximal vital capacity, intra-thoracic gas volume and residual volume: $P<0.001$, total lung capacity: $P=0.006$ ) and showed signs of small airway obstruction (reduced maximum expiratory flow when $75 \%$ of the forced vital capacity (FVC) has been exhaled: $P<0.001$, lesser peak expiratory flow: $P=0.01$ ). There was no significant difference between active and inactive acromegaly. Female patients had significantly altered lung function in terms of subclinical airway obstruction.

Conclusions: In our cross-sectional analysis of lung function in 109 patients with acromegaly, lung volumes were increased compared to healthy controls. Additionally, female patients showed signs of subclinical airway obstruction. There was no difference between patients with active acromegaly compared with patients biochemically in remission.
\end{abstract}

\section{Introduction}

The clinical features of acromegaly affect the function and morphology of multiple organs (1). These changes are associated with clinically relevant comorbidities and an elevated risk of suffering lethal complications, leading to increased mortality compared with the general population (2). It has been shown that patients with uncontrolled acromegaly have their life expectancy reduced by an average of 10 years compared with the general population $(3,4)$. However, the observed-to-expected mortality ratio

www.eje-online.org DOI: 10.1530/EJE-16-1080
() 2017 European Society of Endocrinology Printed in Great Britain appears only to be increased 2- to 4-fold in uncontrolled acromegaly, whereas 'safe' levels of GH and IGF-I are associated with normal mortality rates $(5,6)$.

Based on early research into the causes of death, the increased mortality was believed to be primarily due to cardiovascular events. However, in 1970 Wright et al. showed that respiratory diseases, as well as malignancies, are also noted as the cause of death for number of acromegaly patients (7). Subsequently, other studies

Published by Bioscientifica Ltd. 
have confirmed the high death rate associated with respiratory disorders $(3,8,9,10,11,12)$. Aside from the increased mortality, patients endure an impaired physical performance.

Only few studies have investigated lung function in patients with acromegaly. One major drawback of these previous studies has been the relatively small number of patients examined in each study, ranging from 11 to at most 35 patients. This has led to partly contradicting and inconsistent results. It therefore still remains unclear how lung function parameters in patients with acromegaly differ in comparison to age-, sex- and weight-adjusted healthy controls. We set out to investigate lung function in a large cohort of patients with acromegaly at a single institution.

\section{Subjects and methods}

\section{Patients}

All patients with acromegaly confirmed by OGTT and MRI, who had been treated in our outpatient clinic on a regular basis (Endocrinology, Department of Internal Medicine, Ludwig-Maximilian-University, Munich, Germany), were asked to perform a lung function test in addition to providing a blood sample for laboratory analysis of endocrine and metabolic parameters. Testing took place during a single session. The study was prospectively planned and performed. Due to intermittent shortages of our work force, the study was interrupted several times. All measurements were kept constant throughout the study as much as possible. Patients with previously known severe acute or chronic pulmonary disease or severely impaired cardiac function were excluded from participation $(n=4)$. None of the inquired patients refused to participate in this study. All patients with pituitary deficiencies were on stable hormonal replacement therapy. The local ethics committee granted ethical approval. All study procedures were performed in accordance with the Declaration of Helsinki and all patients gave informed consent.

\section{Laboratory values}

IGF-I and GH samplings were evaluated to determine the biochemical status of patients at the time of lung function testing. IGF-I and GH levels were determined from a single, random blood sample in fasting condition. Regardless of treatment modality all patients with IGF-I levels within age- and gender-adjusted range and with
GH levels less than $1.0 \mu \mathrm{g} / \mathrm{L}$ were considered being in remission (13). As in 22 patients (20.2\%) IGF-I levels were normal, but basal GH levels elevated, we furthermore classified patients according to the clinical course as being clinically in remission.

IGF-I levels were initially measured using a fully automated chemiluminescent immunoassay (Nichols Advantage, Nichols Institute Diagnostics, San Clemente, CA, USA). The linear range was $17-1000 \mathrm{mg} / \mathrm{L}$. Intraassay coefficient of variation (CV) ranged from $11.5 \%$ to $3.5 \%$ at concentrations of $42 \mathrm{ng} / \mathrm{mL}$ and $522 \mathrm{ng} / \mathrm{mL}$ respectively with an inter-assay CV of $10.2-10.6 \%$ (14). The assay was later replaced by the Siemens Immulite 2000 system (Siemens Medical Solutions Diagnostics, Los Angeles, CA, USA) with an analytical sensitivity of $20 \mathrm{ng} /$ $\mathrm{mL}$ with an intra- and inter-assay $\mathrm{CV}<3.9 \%$ and $<8.1 \%$ for IGF-I concentrations between 77 and $1358 \mathrm{ng} / \mathrm{mL}$ (15). To improve IGF-I measurements, the final assay was conducted using the IDS-iSYS system (Immunodiagnostic Systems Ltd.) with a linear range from 10 to $1200 \mathrm{ng} /$ $\mathrm{mL}$, an intra-assay CV of $1.3-3.7 \%$ and an inter-assay CV of $3.4-8.7 \%$ (16). All assays used had good linearity. To adjust for assay differences all IGF-I values are expressed as multiples of the assay-specific age- and sex-adjusted upper limit of normal (defined as 95th percentile of healthy individuals' normal range) in all text, tables and figures.

Serum concentrations of hGH were measured using the automated Advantage chemiluminescent assay system (Nichols Diagnostics Institute, Bad Vilbel, Germany) calibrated against IRP 80/505. This sandwich type immunoassay involves a monoclonal capture- and a polyclonal detection-antibody. Within assay CV was 3.5, 2.2 and $2.9 \%$ at concentrations of $1.4,10.5$ and $28.0 \mathrm{ng} /$ $\mathrm{mL}$ respectively. Between assay variability at the same concentrations was $7.9,2.7$ and $5.9 \%$ respectively. The lower limit of quantification was $0.2 \mathrm{ng} / \mathrm{mL}$, the linear working range was $0.2-50 \mathrm{ng} / \mathrm{mL}$. This assay was later replaced by the Siemens Immulite 2000 chemiluminescent assay system (Siemens) calibrated against IRP 80/505. This sandwich type immunoassay involves a monoclonal mouse-anti-hGH capture- and a polyclonal rabbit-antihGH detection-antibody. Within assay $\mathrm{CV}$ was 3.2, 2.3 and $2.3 \%$ at concentrations of $0.2,2.1$ and $27.1 \mathrm{ng} /$ $\mathrm{mL}$ respectively. Between assay variability at the same concentrations was 3.0, 7.4 and $2.3 \%$ respectively. The lower limit of quantification was $0.05 \mu \mathrm{g} / \mathrm{L}$, the linear working range was $0.05-40 \mu \mathrm{g} / \mathrm{L}$. Finally, a third assay, without pegvisomant cross-reaction, was calibrated with International Standard $98 / 574$ on the IDS-iSYS analyzer. At concentrations between 1.7 and $27.5 \mathrm{ng} / \mathrm{mL}$ 
the intra-assay CV was 2-4\% and inter-assay CV $5-7 \%$. The Immulite and IDS-iSYS assays showed good linear correlation (17).

\section{Lung function test}

All patients performed the lung function test at a single center (Medizinische Klinik und Poliklinik V, Klinikum der Universität München, München, Germany) under standardized conditions. All pulmonary function tests were completed using a Jaeger Masterscope system (Viasys Healthcare, Würzburg, Germany) according to the instructions provided by the manufacturer. Normative data from a large, healthy, non-smoking, Caucasian cohort were available to predict age, sex, weight and height matched reference values (18). Lung function parameters were expressed as percentage of predicted values for descriptive analysis (19), whereas the determined and predicted values were used for comparison between patients with acromegaly and healthy controls (20). Standard spirometric testing included maximal expiratory flow-volume loops, vital capacity (VC), forced expiratory volume in one second (FEV1) and peak expiratory flow (PEF). In order to evaluate small airway function, we also measured maximum expiratory flow when 25, 50 and 75\% respectively of forced VC (FVC) has been exhaled (FEF25, FEF50, FEF75) (21, 22). Airway resistance was assessed using body plethysmography. Residual volume was determined by helium dilution technique. For capillary blood gas analysis, capillary blood was sampled from an earlobe $10 \mathrm{~min}$ after application of a hyperaemizing ointment (Finalgon for blood gas analysis, Boehringer Ingelheim).

\section{Statistical analysis}

Data were analyzed using the Statistical Package for the Social Sciences (IBM SPSS Statistics, version 21, Armonk, NY: IBM). Comparisons of means between groups were calculated using appropriate $t$-tests and effect sizes $(r)$ computed from the $t$-values obtained and the respective degrees of freedom. Effect sizes of 0.1, 0.3 and 0.5 represent small, medium and large effects respectively. Correlation analysis was performed using Pearson's formula where applicable, or Spearman rank correlation when Pearson assumptions were violated. Interval-scaled variables such as duration of disease, laboratory values, and duration of medical treatment were dichotomized by performing a median split in order to perform additional independent t-tests. All testing was two-tailed, and statistical significance was taken at a level of $P \leq 0.05$. Tests with a $P$ value $\leq 0.01$ denote very significant results. A $P$ value $\leq 0.001$ was defined as nominal level of high significance.

\section{Results}

A total of 109 acromegaly patients participated in this cross-sectional study. Table 1 summarizes the clinical characteristics of the study population. At the time of testing a total of 80 patients had biochemically active acromegaly following strict criteria of cure. Of these, 20 patients were newly diagnosed. The remaining 60 patients had unsuccessful pituitary surgery in 50 cases (83\%) and 14 patients had pituitary irradiation in their past $(23 \%$, years since irradiation: $11.9 \pm 8.3)$. 36 patients $(60 \%)$ received pharmacological treatment of GH excess. Most patients with active acromegaly were tested after surgery before starting medical treatment $(n=17,71 \%)$. All 29 patients in remission had neurosurgical intervention in their past and 7 patients were also irradiated (24\%). 10 patients had to continue GH suppressing medical treatment. Spirometry including flow-volume loops, body plethysmography and blood gas analysis were performed without any complications.

The comparison of predicted mean values from age, sex, weight and height matched healthy adults to measured values in our acromegaly patients yielded several significant differences regarding lung volumes as well as parameters of obstruction. Acromegaly patients had a greater lung volume as measured by maximal vital capacity (VCmax; $P<0.001, r=0.5$ ), intra-thoracic gas volume (ITGV; $P<0.001, r=0.48$ ), total lung capacity (TLC; $P=0.006, r=0.26)$ and residual volume (RV; $P<0.001$, $r=0.49$ ). In $40.4 \%$ of patients, the increase in residual volume was clinically relevant (i.e. more than $120 \%$ of predicted value).

There were also changes hinting at airway obstruction, with a significantly smaller PEF ( $P=0.01, R=0.24)$ noted in acromegaly patients, in addition to reduced maximum expiratory flow when $75 \%$ of the FVC has been exhaled (FEF75; $P<0.001$ ). A clinically relevant reduction (i.e. less than $80 \%$ of predicted value) in FEF75 was found in $56.5 \%$ of our patients. However, total specific resistance (sRtot) was reduced $(P=0.005, r=0.27)$ and forced expiratory volume in $1 \mathrm{~s}$ (FEV1) was elevated $(P<0.001, r=0.43)$. No other significant differences in spirometry and body plethysmography were observed between the healthy adult data and our study cohort. 
Table 1 Clinical characteristics of the study population. Data are presented as mean \pm S.D.

\begin{tabular}{l}
\hline Characteristics \\
\hline Age \\
Random GH levels $(\mathrm{ng} / \mathrm{mL})$ \\
Newly diagnosed $(n=20)$ \\
Active, currently not treated $(n=24)$ \\
Active despite med. treatment $(n=36)$ \\
Inactive with med. treatment $(n=10)$ \\
In remission w/o further treatment $(n=19)$ \\
IGF-I/ULN \\
Newly diagnosed ( $n=20)$ \\
Active, currently not treated $(n=24)$ \\
Active despite med. treatment $(n=36)$ \\
Inactive with med. treatment $(n=10)$ \\
In remission w/o further treatment $(n=19)$ \\
Delay in diagnosis (years from first symptoms to diagnosis) \\
Years since diagnosis \\
History of pituitary surgery \\
History of pituitary irradiation \\
Pituitary deficiency \\
Thyroid axis \\
Gonadal axis \\
Adrenal axis \\
Smokers \\
Years in biochemical remission
\end{tabular}

\begin{tabular}{c}
\hline Total $(n=109)$ \\
\hline $54.6 \pm 13.5$ \\
$7.1 \pm 16.8$ \\
$23.5 \pm 31.7$ \\
$5.5 \pm 9.3$ \\
$4.6 \pm 8.3$ \\
$0.5 \pm 0.3$ \\
$0.4 \pm 0.3$ \\
$1.36 \pm 0.87$ \\
$2.62 \pm 0.92$ \\
$1.32 \pm 0.67$ \\
$1.24 \pm 0.45$ \\
$0.78 \pm 0.18$ \\
$0.62 \pm 0.23$ \\
$5.2 \pm 5.9$ \\
$12.7 \pm 11.6$ \\
$79(72.5 \%)$ \\
$21(19.3 \%)$ \\
$56(51.4 \%)$ \\
$26(23.9 \%)$ \\
$47(43.1 \%)$ \\
$36(33.0 \%)$ \\
$8(7.3 \%)$ \\
$10.5 \pm 9.1(n=29)$ \\
\end{tabular}

\begin{tabular}{ccc}
\hline Male patients $(n=53,48.6 \%)$ & & Female patients $(n=56,51.4 \%)$ \\
\cline { 1 - 1 } $50.6 \pm 12.6$ & & $58.4 \pm 13.4$ \\
$6.5 \pm 17.5$ & $7.8 \pm 16.2$ \\
$25.1 \pm 47.1$ & $22.8 \pm 24.7$ \\
$9.9 \pm 12.5$ & $1.9 \pm 1.7$ \\
$3.9 \pm 4.3$ & $5.5 \pm 11.6$ \\
$0.4 \pm 0.2$ & $0.7 \pm 0.2$ \\
$0.4 \pm 0.3$ & $0.4 \pm 0.2$ \\
$1.35 \pm 0.69$ & $1.37 \pm 1.01$ \\
$2.23 \pm 0.71$ & $2.79 \pm 0.97$ \\
$1.54 \pm 0.82$ & $1.13 \pm 0.47$ \\
$1.49 \pm 0.42$ & $0.92 \pm 0.26$ \\
$0.77 \pm 0.23$ & $0.80 \pm 0.11$ \\
$0.70 \pm 0.18$ & $0.53 \pm 0.25$ \\
$5.1 \pm 4.7$ & $5.3 \pm 6.9$ \\
$12.8 \pm 10.6$ & $12.6 \pm 12.6$ \\
$41(77.4 \%)$ & $38(67.9 \%)$ \\
$12(22.6 \%)$ & $9(16.1 \%)$ \\
$31(58.5 \%)$ & $25(44.6 \%)$ \\
$13(24.5 \%)$ & $13(23.2 \%)$ \\
$31(58.5 \%)$ & $16(28.6 \%)$ \\
$19(35.8 \%)$ & $17(30.4 \%)$ \\
$4(7.5 \%)$ & $4(7.1 \%)$ \\
$10.0 \pm 10.3(n=16)$ & $11.2 \pm 7.7(n=13)$ \\
&
\end{tabular}

Blood gas analysis showed significant hypoxemia in acromegaly patients when compared to normative data (pO2; $P<0.001, r=0.56)$, with a mean $\mathrm{pO} 2$ of $93.3 \% \pm 10.0 \%$ of the predicted value, and mean pCO2 levels $102.1 \% \pm 9.9 \%$ of predicted value. However, only 6 of our patients $(5.6 \%)$ had clinically relevant hypoxemia. Of these, 4 patients were obese (1 with body mass index, BMI $\geq 35 \mathrm{~kg} / \mathrm{m}^{2}$, 3 with BMI $\geq 30 \mathrm{~kg} / \mathrm{m}^{2}$ ). Figure 1 summarizes the lung function parameters of the study population as percentage of predicted values.

Sub-group analysis showed no significant difference in lung function between active and inactive acromegaly (cf. Supplementary Table 3 for tabular overview, see section on supplementary data given at the end of this article). Further, after splitting GH and IGF-I values into tertiles, no statistically significant differences were observed between patients with low levels of GH or IGF when compared to those with high levels. Neither time from first symptoms to diagnosis, nor duration of disease correlated with lung function. For those patients in remission, time in remission was positively correlated with VCmax $(r=0.37, P=0.045)$ and FEF25 $(r=0.44, P=0.016)$. Patients with shorter time in remission (up to 4 years) had a mean VCmax of $102.2 \% \pm 20.3 \%$ as compared to $117.7 \% \pm 13.0 \%$ in patients with longer disease remission (4-40 years). Similarly, FEF25 was $78.6 \% \pm 22.8 \%$ and $104.3 \% \pm 24.1 \%$ respectively; cf. Fig. 2.
There was no significant correlation between duration of medical treatment with somatostatin analogs (SSA) and lung function; nonetheless, comparison of patients who have received SSA for up to 4 years with patients who have had SSA for a longer period of time (4-26 years), showed significant differences between the two groups

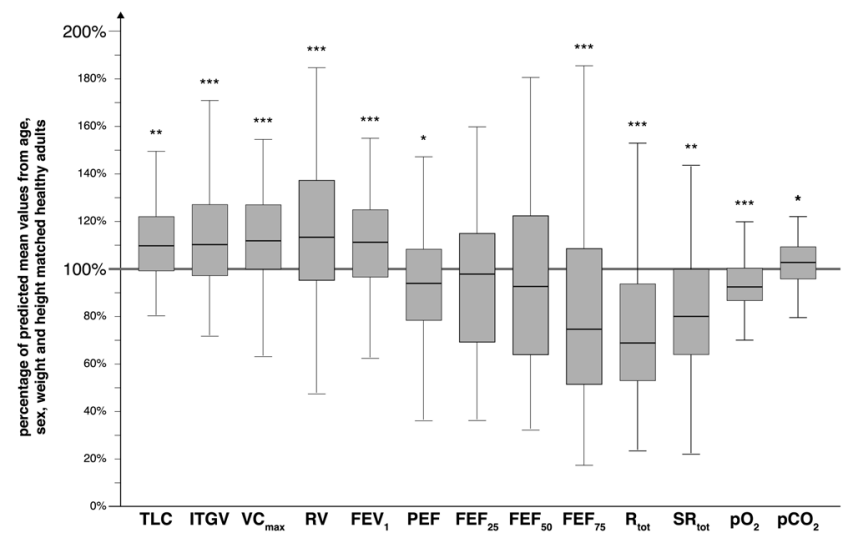

\section{Figure 1}

Box-plots of lung function parameters in acromegaly patients relative to predicted values. Lung volumes are increased in patients with acromegaly. They also show signs of airway obstruction and hypoxemia. All parameters are expressed as percentage of predicted mean values from age, sex, weight and height matched healthy adults. Significant differences at $P<0.001$ are marked by ***, $P<0.01$ by ** and $P<0.05$ by *. 


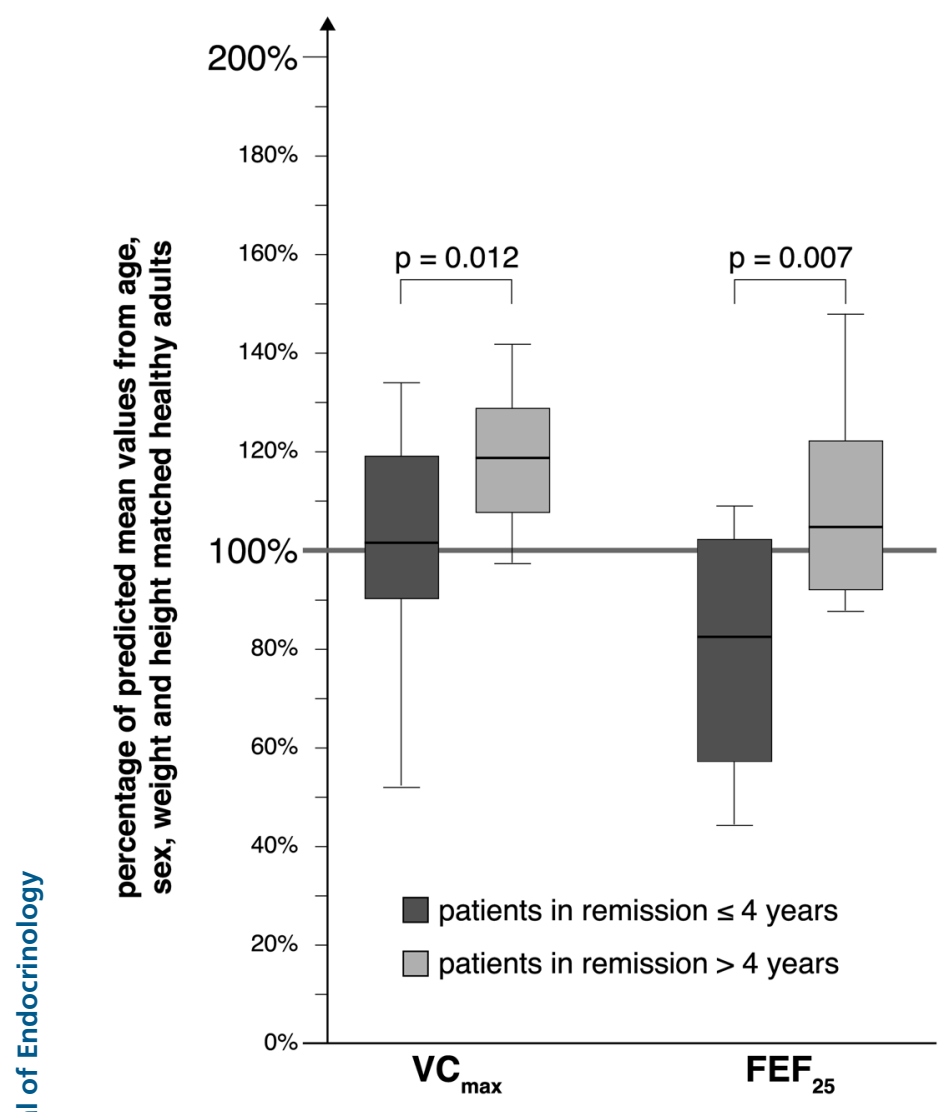

negatively correlated with ITGV $(r=-0.29, P=0.002)$, RV $(r=-0.19, P=0.046)$ and pO2 $(r=-0.31, P=0.001)$. Other lung function parameters were not affected by BMI.

Patients who smoke had a significantly lower relative forced expiratory volume in one second (FEV1/VC; $P=0.011, r=0.24)$ and larger TLC $(P=0.049, r=0.19)$. All other lung function parameters did not significantly differ between smoking and non-smoking patients. Post hoc exclusion of smoking patients from the dataset did not alter the findings presented above.

\section{Discussion}

The present study assessed lung function in a large cohort of 109 patients with acromegaly at an outpatient clinic. As expected, a majority of patients with acromegaly have lung overgrowth with both increased lung volume, and increased residual volume as shown in our study as well as numerous other studies before $(23,24,25,26,27,28)$. The cause for the increase in lung size has been subject of much debate: Aside from hypertrophy of interstitial tissue (26), an increase in the number of alveoli (30), an

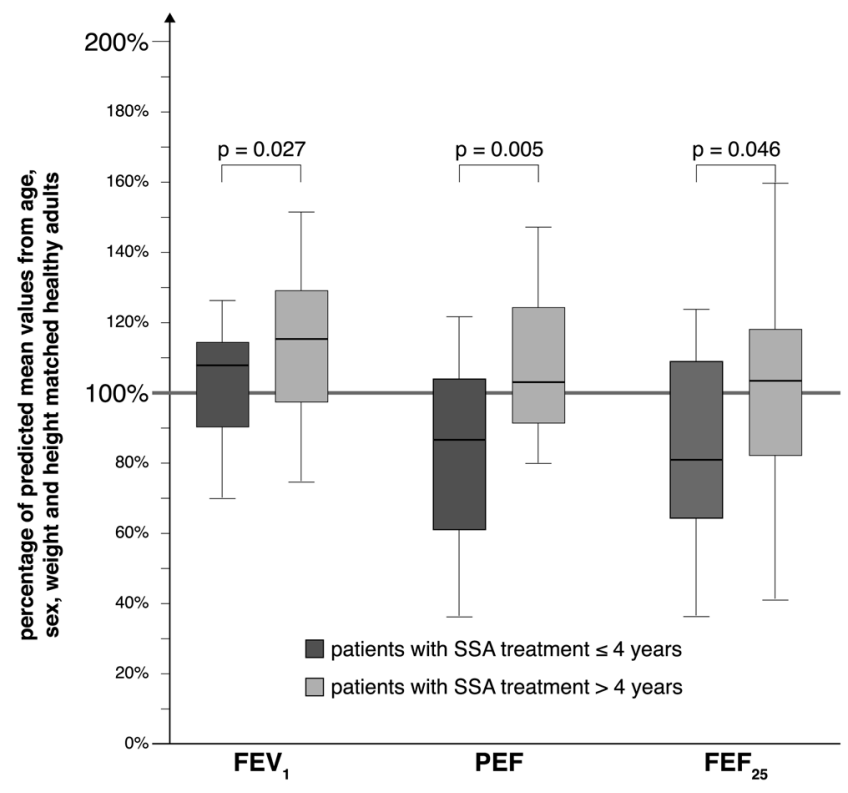

Figure 2

Differences of lung function parameters depending on duration of remission of acromegaly. VCmax and FEF25 respectively differ between patients with shorter duration of remission ( $\leq 4$ years) from their acromegaly compared to patients with longer disease remission. All parameters are expressed as percentage of predicted mean values from age, sex, weight and height matched healthy adults.

vis-à-vis FEV1 $(P=0.027, r=0.32)$, PEF $(P=0.005, r=0.41)$ and FEF25 $(P=0.046, r=0.29)$. Mean percentage of predicted values were $102.8 \% \pm 16.1 \%$ vs $116.0 \% \pm 22.5 \%$ (FEV1), $82.8 \% \pm 24.5 \%$ vs $105.9 \% \pm 28.1 \%$ (PEF) and $83.1 \% \pm 27.3 \%$ vs $101.1 \% \pm 31.9 \%$ (FEF25) in normative data and acromegaly patients respectively, cf. Fig. 3.

Comparison of lung function of male and female patients with acromegaly respectively revealed statistically significant differences in lung parameters associated with airway obstruction. Male patients had significantly higher flow rates concerning PEF $(P=0.013, r=0.24)$, FEF25 $(P=0.003, r=0.28)$ and FEF50 $(P=0.001, r=0.31)$. Total airway resistance (Rtot) was lower in men than women $(P=0.005, r=0.27)$, but was not elevated in either sex. Table 2 gives an overview of lung function in male and female patients with acromegaly. BMI of patients was

\section{Figure 3}

Differences of lung function parameters depending on duration of medical treatment with somatostatin analogs (SSA). FEV1, PEF and FEF25 respectively differ between patients with shorter duration of medical treatment with somatostatin analogs ( $\leq 4$ years) compared to patients with longer treatment duration. All parameters are expressed as percentage of predicted mean values from age, sex, weight and height matched healthy adults. 
Table 2 Differences in lung function between male and female patients with acromegaly.

\begin{tabular}{lcc} 
& & Male \\
\hline PEF & & $98.9 \% \pm 27.9 \%$ \\
FEF25 & $103.2 \% \pm 28.9 \%$ \\
FEF50 & $103.9 \% \pm 34.8 \%$ \\
sRtot & $80.3 \% \pm 45.7 \%$ \\
Rtot $(\mathrm{kPa} * \mathrm{~s} / \mathrm{L})$ reference range: $<0.3$ & $0.2 \pm 0.09$ \\
\hline
\end{tabular}

\begin{tabular}{c}
\hline Female \\
\hline $86.3 \% \pm 21.3 \%$ \\
$86.6 \% \pm 27.6 \%$ \\
$82.6 \% \pm 30.8 \%$ \\
$97.4 \% \pm 47.5 \%$ \\
$0.26 \pm 0.12$ \\
\hline
\end{tabular}

\begin{tabular}{ll}
\hline \multicolumn{1}{c}{$\boldsymbol{P}$} \\
\hline 0.013 \\
0.003 \\
0.001 \\
0.06 \\
0.005 \\
\hline
\end{tabular}

\begin{tabular}{c}
\hline Effect size \\
\hline 0.24 \\
0.28 \\
0.31 \\
0.18 \\
0.27
\end{tabular}

Effect size $(r)$ is calculated from the $t$-values obtained and the respective degrees of freedom.

FEF25, maximum expiratory flow when $25 \%$ of the FVC has been exhaled; FEF50, maximum expiratory flow when $50 \%$ of the FVC has been exhaled; PEF, peak expiratory flow; Rtot, total airway resistance.

increase in alveolar size $(27,31,32)$ as well as air trapping (31) has been discussed. Our data show increased lung volumes in men and women alike without statistical difference. In 1326 healthy subjects from the Study of Health in Pomerania (SHIP), a positive correlation was seen between IGF-I, IGF-I/IGFBP-3 ratio and FVC in men of all ages. In women this correlation was only present in post-menopausal women over the age of 50 and explained by differences in sexual hormone levels and their effect on the GH/IGF-I axis (29). In contrast to the healthy individuals in SHIP all of our patients suffered from acromegaly and thus at some point in time increased GH/ IGF-I levels regardless of menopause. As female patients with a deficiency of the gonadotropic axis were on stable hormone replacement (data not shown), a sex hormone deficiency is unlikely the cause of our findings. We assume that excess of growth hormone in active acromegaly overcompensates for any regulatory action on pulmonary morphology of sex hormones in women. In patients with acromegaly Brody et al. failed to show increased lung size in women altogether, but only 4 female patients were included in that study (26).

We detected a significant decrease in PEF as well as maximum expiratory flow at $75 \%$ of FVC (FEF75). The latter indicates small airway disease in acromegaly patients. Overgrowth of pulmonary epithelium and thickening of interstitial tissue is a consequence of proliferation of pneumocytes and smooth muscle cells. This leads to decreased elasticity of the lung (1). Furthermore, as the thoracic compartment is unable to give the growing lung tissue the necessary room, obstruction of the small airways eventually results. This in turn can lead to emphysema, and potentially to air trapping. Conversely, forced expiratory volume in one second (FEV1), a sign of large airway obstruction, is elevated in our patients in comparison to normative data. Essentially, this means that in our cohort we found signs of probable mild small airway disease that has yet to impact parameters associated with large airway disease (33). Previous studies have demonstrated narrowing of both small (27), and upper airways $(23,24,25,27)$. Others have not shown any signs of obstruction (26).

FEF25 normalized in our patients with longer disease remission, hinting at the possibility of reversal of morphological and obstructive changes over years or even decades. However, in our data these changes were not reflected by other parameters of obstruction, which may be a limitation of the cross-sectional nature of our study.

We saw normalization of FEV1, PEF and FEF25 in patients with long-lasting somatostatin analog (SSA) treatment. Few studies have shown beneficial effects of octreotide in sleep apnea, but changes to the respiratory tract are still largely deemed permanent (35). As somatostatin receptors subtypes 1 through 5 (SSTR1-5) are expressed in bronchial glands (36), and others including SSTR2 are expressed in interstitial lung tissue, SSA are currently being investigated as agent in pulmonary fibrosis (37). It seems possible that long-term application of SSA in acromegaly might reverse pathologic changes in lung function through a primary effect on lung tissue.

In our analysis, we found that disease activity in acromegaly is not associated with changes in lung function parameters. This was equally true when comparing only newly diagnosed patients with patients in remission (data not shown). Prior studies looking into lung function in patients with acromegaly have only shown findings indicative of an association between disease activity and lung function parameters and remained inconclusive. Like others before us $(24,32,34)$ we could not show that disease duration significantly increases the risk of developing respiratory abnormalities as has been previously reported $(23,27)$.

We found subclinical hypoxemia with arterial blood tension ranging from 55 to $111 \mathrm{mmHg}$ in our patients which has previously been shown by Luboshitzky et al. (25). Only 6 patients in our cohort (5.6\%) had clinically relevant hypoxemia. Of these, 4 patients were obese, which might explain the severity of hypoxemia in these patients. Other investigations failed to demonstrate a mismatch of ventilation/perfusion in acromegaly (1). 
It has been shown that patients with active acromegaly have increased non-aerated and poorly aerated compartments (28).

Smoking had no effect on the main findings of this study. The few smoking patients in our cohort showed increased TLC and reduced FEV1. While the latter has been well shown to be due to the loss of lung elastic recoil pressure (38), the effect of smoking on TLC has been a matter of debate (39). Notably, the fraction of smokers in our cohort $(7.3 \%)$ is very small compared to that of the general population in Bavaria (23.9\%) (40), but is in line with other recent studies of acromegaly where smoking habits were disclosed $(41,42)$. Therefore, due to the small numbers, caution should be taken when interpreting these results.

There are a few limitations to our study: A crosssectional study requires careful interpretation of results as it does not allow for inference of causal relationships possible when longitudinal data are available. It may be argued that comparison with actual healthy controls, rather than predicted normative data may have been beneficial. This is only partly true, as normative data accurately reflect healthy controls. However, testing against patients with growth hormone deficiency could have strengthened the results.

It is noteworthy that although the number of active acromegaly patients in our cohort is fairly large, the number of patients considered biochemically in remission is unusually small. This is attributable to the strict criteria of cure used to determine the patients' status, which led to a higher number of patients considered 'active', e.g. when random GH levels failed to meet the $1 \mathrm{ng} / \mathrm{mL}$ criterion. This is especially true in our case, since the assays used to measure GH and IGF-I in our laboratory changed during the course of the study. This may lead to overestimation of patients with active disease, increasing the potential for type II errors due to incorrect assumptions in the data. However, we tested the possibility of overlooking an effect of disease activity by coding for 'clinically in remission', which reflected whether or not a patient was considered in stable remission by looking through the patient's history (either with or without continuing medical treatment). This did not yield any significant results (data not shown).

To our knowledge this is the largest series of lung function assessment in acromegaly patients to date. Sample sizes of previous studies range from 11 to 35 patients. With a sample size of 109 patients, more than threefold the largest previous study, we were able to detect subtle effects and could perform sub-group analysis to elucidate points previously associated with mixed results.
We believe that our data are a strong foundation for further research that may focus on the dynamics of active disease on lung function and potentially the effects of treatment and remission.

\section{Conclusion}

In our cross-sectional analysis we could show that lung volumes of patients with acromegaly are significantly increased compared to healthy controls. Also, patients are hypoxemic and show signs of airway obstruction in terms of reduced PEF and lower maximum expiratory flow when $75 \%$ of the FVC has been exhaled. Airway obstruction is more pronounced in female patients. There was no difference between patients with active acromegaly compared to patients who are biochemically in remission. Patients with long-standing remission had increased VC as compared to patients with shorter time in remission.

\section{Supplementary data}

This is linked to the online version of the paper at http://dx.doi.org/10.1530/ EJE-16-1080.

\section{Declaration of interest}

The authors declare that there is no conflict of interest that could be perceived as prejudicing the impartiality of this study.

\section{Funding}

This research did not receive any specific grant from any funding agency in the public, commercial or not-for-profit sector.

\section{References}

1 Colao A, Ferone D, Marzullo P \& Lombardi G. Systemic complications of acromegaly: epidemiology, pathogenesis, and management. Endocrine Reviews 200425 102-152. (doi:10.1210/er.2002-0022)

2 Dekkers OM, Biermasz NR, Pereira AM, Romijn JA \& Vandenbroucke JP. Mortality in acromegaly: a metaanalysis. Journal of Clinical Endocrinology and Metabolism 200893 61-67. (doi:10.1210/ jc.2007-1191)

3 Rajasoorya C, Holdaway IM, Wrightson P, Scott DJ \& Ibbertson HK. Determinants of clinical outcome and survival in acromegaly. Clinical Endocrinology 199441 95-102. (doi:10.1111/j.1365-2265.1994. tb03789.x)

4 Holdaway IM. Factors influencing mortality in acromegaly. Journal of Clinical Endocrinology and Metabolism 200489 667-674. (doi:10.1210/ jc.2003-031199)

5 Maison P, Tropeano AI, Macquin-Mavier I, Giustina A \& Chanson P. Impact of somatostatin analogs on the heart in acromegaly: a metaanalysis. Journal of Clinical Endocrinology and Metabolism 200792 1743-1747. (doi:10.1210/jc.2006-2547)

6 Kauppinen-Mäkelin R, Sane T, Reunanen A, Välimäki MJ, Niskanen L, Markkanen H, Löyttyniemi E, Ebeling T, Jaatinen P, Laine H et al. A nationwide survey of mortality in acromegaly. Journal of Clinical 
Endocrinology and Metabolism 200590 4081-4086. (doi:10.1210/ jc.2004-1381)

7 Wright AD, Hill DM, Lowy C \& Fraser TR. Mortality in acromegaly. Quarterly Journal of Medicine 197039 1-16. (doi:10.1093/ oxfordjournals.qjmed.a067196)

8 Alexander L, Appleton D, Hall R, Ross WM \& Wilkinson R. Epidemiology of acromegaly in the Newcastle region. Clinical Endocrinology 198012 71-79. (doi:10.1111/j.1365-2265.1980. tb03135.x)

9 Etxabe J, Gaztambide S, Latorre P \& Vazquez JA. Acromegaly: an epidemiological study. Journal of Endocrinological Investigation 199316 181-187. (doi:10.1007/BF03344942)

10 Bates AS, Van't Hoff W, Jones JM \& Clayton RN. An audit of outcome of treatment in acromegaly. Quarterly Journal of Medicine $1993 \mathbf{8 6}$ 293-299. (doi:10.1093/oxfordjournals.qjmed.a068816)

11 Orme SM, McNally RJ, Cartwright RA \& Belchetz PE. Mortality and cancer incidence in acromegaly: a retrospective cohort study. United Kingdom Acromegaly Study Group. Journal of Clinical Endocrinology and Metabolism 199883 2730-2734. (doi:10.1210/jc.83.8.2730)

12 Sherlock M, Reulen RC, Aragon-Alonso A, Ayuk J, Clayton RN, Sheppard MC, Hawkins MM, Bates AS, \& Stewart PM. A paradigm shift in the monitoring of patients with acromegaly: last available growth hormone may overestimate risk. Journal of Clinical Endocrinology and Metabolism 201499 478-485. (doi:10.1210/jc.20132450)

13 Giustina A, Chanson P, Bronstein MD, Klibanski A, Lamberts S, Casanueva FF, Trainer P, Ghigo E, Ho K \& Melmed S. A consensus on criteria for cure of acromegaly. Journal of Clinical Endocrinology and Metabolism 201095 3141-3148. (doi:10.1210/jc.2009-2670)

14 Brabant G, zur Mühlen A, Wüster C, Ranke MB, Kratzsch J, Kiess W, Ketelslegers JM, Wilhelmsen L, Hulthén L, Saller B et al. Serum insulin-like growth factor I reference values for an automated chemiluminescence immunoassay system: results from a multicenter study. Hormone Research 200360 53-60. (doi:10.1159/000071871)

15 Elmlinger MW, Kühnel W, Weber MM \& Ranke MB. Reference ranges for two automated chemiluminescent assays for serum insulin-like growth factor I (IGF-I) and IGF-binding protein 3 (IGFBP-3). Clinical Chemistry and Laboratory Medicine 200442 654-664. (doi:10.1515/ CCLM.2004.112)

16 Bidlingmaier M, Friedrich N, Emeny RT, Spranger J, Wolthers OD, Roswall J, Koerner A, Obermayer-Pietsch B, Hübener C, Dahlgren $\mathrm{J}$ et al. Reference intervals for insulin-like growth factor-1 (IGF-1) from birth to senescence: results from a multicenter study using a new automated chemiluminescence igf- 1 immunoassay conforming to recent international recommendations. Journal of Clinical Endocrinology and Metabolism 201499 1712-1721. (doi:10.1210/ jc.2013-3059)

17 Manolopoulou J, Alami Y, Petersenn S, Schopohl J, Wu Z, Strasburger CJ \& Bidlingmaier M. Automated 22-kD growth hormone-specific assay without interference from Pegvisomant. Clinical Chemistry 2012 58 1446-1456. (doi:10.1373/clinchem.2012.188128)

18 Quanjer PH, Tammeling GJ, Cotes JE, Pedersen OF, Peslin R \& Yernault JC. Lung volumes and forced ventilatory flows. European Respiratory Journal 19936 (Supplement 16) 5-40. (doi:10.1183/090419 50.005s1693)

19 Morris JF. Spirometry in the evaluation of pulmonary function. Western Journal of Medicine 1976125 110-118.

20 Crapo RO. The role of reference values in interpreting lung function tests. European Respiratory Journal 200424 341-342. (doi:10.1183/0903 1936.04.00063804)

21 Cochrane GM, Benatar SR, Davis J, Collins J V, \& Clark TJ. Correlation between tests of small airway function. Thorax 197429 172-178. (doi:10.1136/thx.29.2.172)

22 Cochrane GM, Prieto F, Hickey B, Benatar SR \& Clark TJ. Early diagnosis of airways obstruction. Thorax 197429 389-393. (doi:10.1136/thx.29.4.389)
23 Evans CC, Hipkin LJ, \& Murray GM. Pulmonary function in acromegaly. Thorax 197732 322-327. (doi:10.1136/thx.32.3.322)

24 Trotman-Dickenson B, Weetman AP \& Hughes JM. Upper airflow obstruction and pulmonary function in acromegaly: relationship to disease activity. Quarterly Journal of Medicine 199179 527-538. (doi:10.1136/bmj.302.6775.527)

25 Luboshitzky R \& Barzilai D. Hypoxemia and pulmonary function in acromegaly. American Review of Respiratory Disease 1980121 471-475. (doi:10.1164/arrd.1980.121.3.471)

26 Brody JS, Fisher AB, Gocmen A \& DuBois AB. Acromegalic pneumonomegaly: lung growth in the adult. Journal of Clinical Investigation 197049 1051-1060. (doi:10.1172/JCI106321)

27 Harrison BD, Millhouse KA, Harrington M \& Nabarro JD. Lung function in acromegaly. Quarterly Journal of Medicine $1978 \mathbf{4 7}$ 517-532. (doi:10.1093/oxfordjournals.qjmed.a067556)

28 Camilo GB, Carvalho ARS, Machado DC, Mogami R, Melo PL \& Lopes AJ. CT pulmonary densitovolumetry in patients with acromegaly: a comparison between active disease and controlled disease. British Journal of Radiology 201588 20150315. (doi:10.1259/ bjr.20150315)

29 Gläser S, Friedrich N, Ewert R, Schäper C, Nauck M, Dörr M, Völzke H, Felix SB, Krebs A, Wallaschofski $\mathrm{H}$ et al. Association between serum insulin-like growth factor (IGF) I and IGF binding protein-3 and lung function. Journal of Clinical Endocrinology and Metabolism 200994 2452-2458. (doi:10.1210/jc.2008-2662)

30 Donnelly PM, Grunstein RR, Peat JK, Woolcock AJ \& Bye PT. Large lungs and growth hormone: an increased alveolar number? European Respiratory Journal 19958 938-947. (doi:10.1183/ 09031936.95.08060938)

31 Camilo GB, Guimarães FS, Silva DPG, Mogami R, Kasuki L, Gadelha MR, Melo PL \& Lopes AJ. Pulmonary function testing and chest tomography in patients with acromegaly. Multidisciplinary Respiratory Medicine 20138 70. (doi:10.1186/2049-6958-8-70)

32 García-Río F, Pino JM, Díez JJ, Ruíz A, Villasante C \& Villamor J. Reduction of lung distensibility in acromegaly after suppression of growth hormone hypersecretion. American Journal of Respiratory and Critical Care Medicine 2001164 852-857. (doi:10.1164/ ajrccm.164.5.2005059)

33 McNulty W \& Usmani OS. Techniques of assessing small airways dysfunction. European Clinical Respiratory Journal 20141 1-17. (doi:10.3402/ecrj.v1.25898)

34 Benfante A, Ciresi A, Bellia M, Cannizzaro F, Bellia V, Giordano C \& Scichilone N. Early lung function abnormalities in acromegaly. Lung 2015193 393-399. (doi:10.1007/s00408-015-9710-1)

35 Colao A, Auriemma RS, Pivonello R, Galdiero M, \& Lombardi G. Medical consequences of acromegaly: what are the effects of biochemical control? Reviews in Endocrine and Metabolic Disorders 2008 9 21-31. (doi:10.1007/s11154-007-9062-0)

36 Taniyama Y, Suzuki T, Mikami Y, Moriya T, Satomi S \& Sasano H. Systemic distribution of somatostatin receptor subtypes in human: an immunohistochemical study. Endocrine Journal 200552 605-611. (doi:10.1507/endocrj.52.605)

37 Egger C, Gérard C, Vidotto N, Accart N, Cannet C, Dunbar A, Tigani B, Piaia A, Jarai G, Jarman E et al. Lung volume quantified by MRI reflects extracellular-matrix deposition and altered pulmonary function in bleomycin models of fibrosis: effects of SOM230. American Journal of Physiology: Lung Cellular and Molecular Physiology 2014306 L1064-L1077. (doi:10.1152/ajplung.00027.2014)

38 Hogg JC, Wright JL, Wiggs BR, Coxson HO, Opazo Saez A \& Paré PD Lung structure and function in cigarette smokers. Thorax 199449 473-478. (doi:10.1136/thx.49.5.473)

39 Miller GJ. Cigarette smoking and irreversible airways obstruction in the West Indies. Thorax 197429 495-504. (doi:10.1136/thx.29.5.495)

40 Stang A \& Stang M. An inter-state comparison of cardiovascular risk factors in Germany. Deutsches Ärzteblatt International 2014111 530-536. (doi:10.3238/arztebl.2014.0530) 
41 Fadini GP, Dassie F, Albiero M, Boscaro E, Albano I, Martini C, Vigili de Kreutzenberg S, Agostini C, Avogaro A, Vettor R et al. Endothelial progenitor cells are reduced in acromegalic patients and can be restored by treatment with somatostatin analogues. Journal of Clinical Endocrinology and Metabolism 201499 E2549-E2556. (doi:10.1210/jc.2014-2275)
42 Svensson J, Carlzon D, Petzold M, Karlsson MK, Ljunggren O,

Tivesten A, Mellström D \& Ohlsson C. Both low and high serum igf-I levels associate with cancer mortality in older men. Journal of Clinical Endocrinology and Metabolism 201297 4623-4630. (doi:10.1210/ jc.2012-2329)

Received 30 December 2016

Revised version received 18 April 2017

Accepted 21 April 2017 